\title{
ARTICLE Synergistic antitumor activity of sorafenib and artesunate in hepatocellular carcinoma cells
}

\author{
Xu Yao ${ }^{1}$, Chen-ru Zhao ${ }^{1}$, Hao Yin ${ }^{1}$, KeWei Wang ${ }^{1}$ and Jian-jun Gao ${ }^{1}$
}

Sorafenib is currently the standard chemotherapy drug for treatment of advanced hepatocellular carcinoma (HCC). But its efficacy requires improvement, it is imperative to seek therapeutic strategies that combine sorafenib with other anticancer agents. In this study we investigated the synergistic anticancer effect of combining sorafenib and artesunate, an anti-malaria drug derivative, against HCC in vitro and in vivo. We first showed that artesunate $(1-100 \mu \mathrm{M})$ alone dose-dependently inhibited the proliferation of five HCC cell lines tested with $\mathrm{IC}_{50}$ values of around $100 \mu \mathrm{M}$. Artesunate treatment dose-dependently increased the ROS level in both HuH7 and Hep3B cells; addition of NAC significantly ameliorated the antiproliferation effect of artesunate against HuH7 and Hep3B cells. Then we demonstrated that combination of sorafenib and artesunate exerted synergistic antiproliferation effect and induced synergistic apoptosis in HCC cell lines. In nude mice bearing Hep3B xenografts, combined administration of sorafenib and artesunate significantly enhanced the suppression on tumor growth. We further revealed that sorafenib dose-dependently decreased the levels of p-ERK and p-STAT3, whereas artesunate markedly increased the levels of p-ERK and p-STAT3 in HuH7 and Hep3B cells. When used in combination, sorafenib abolished artesunate-elevated levels of p-STAT3 and p-ERK. Moreover, pharmacological inhibition of ERK by inhibitor PD0325901 or STAT3 by inhibitor Stattic markedly enhanced the anticancer activity of artesunate, suggesting that suppression of ERK and STAT3 signaling by sorafenib contributes to the synergistic anticancer activity against HCC caused by combination of sorafenib and artesunate. Taken together, our results provide an evidence for possible use of sorafenib plus artesunate or artemisinin analogs for treatment of HCC in the future.

Keywords: sorafenib; ERK; artemisinin; ROS; hepatocellular carcinoma; STAT3

Acta Pharmacologica Sinica (2020) 41:1609-1620; https://doi.org/10.1038/s41401-020-0395-5

\section{INTRODUCTION}

Hepatocellular carcinoma (HCC) is one of the most common solid cancers and a leading cause of cancer-related death worldwide [1-3]. China currently accounts for over half of the global incidence of HCC due to the high rate of chronic hepatitis B virus infection $[4,5]$. HCC exhibits low sensitivity to chemotherapeutic agents, and there are currently limited drugs available for disease treatment $[6,7]$. Sorafenib, an inhibitor of the nonreceptor tyrosine kinase RAF and several receptor tyrosine kinases, is a first-line and widely used drug for the systemic treatment of advanced-stage HCC [8]. However, only $\sim 30 \%$ of patients are responsive to sorafenib, and the median overall survival is only prolonged by 2.8 months $[9,10]$. Therefore, it is imperative to improve the efficacy of sorafenib for the improved treatment of HCC.

Artesunate, derived from artemisinin, is a first-line drug used for the treatment of severe malaria in adults and children with a welltolerated safety profile [11]. In addition to its therapeutic effect in malaria, many studies have demonstrated that artesunate also exerts broad anticancer activity by suppressing cell proliferation and angiogenesis and triggering apoptosis in vitro and in vivo [12-16]. Clinical trials are currently in progress to test the efficacy of artesunate against several types of cancers, including bowel cancer, breast cancer, colorectal cancer, and HCC [17]. However, whether combining sorafenib and artesunate can produce synergistic effects on anticancer activity remains to be investigated.

Artesunate is a prodrug that is rapidly converted to its active form dihydroartemisinin (DHA) via plasma esterase enzyme [18]. It is hypothesized that the cleavage of the endoperoxide bridge in the pharmacophore of DHA generates reactive oxygen species (ROS), which increases oxidative stress and causes cancer cell death [19]. We previously found that the ROS-generated agent capsaicin synergistically enhances the anticancer activity of sorafenib in HCC by activating ERK signaling [20]. Enlightened by this finding, we attempted to test the activity of artesunate plus sorafenib against HCC cells.

In this study, we show that artesunate alone dose-dependently suppressed the proliferation of HCC cells. Combining artesunate and sorafenib achieved synergistic effects on suppressing cell proliferation and cell apoptosis induction. Artesunate induced increased levels of ROS in HCC cells, thus leading to an increased Bax/Bcl-2 level and cell apoptosis. Mechanistically, we found that artesunate activates ERK and STAT3 signaling, which is attenuated by sorafenib in HCC cells. Our findings may help explain the synergistic suppression of HCC cells by the combined use of artesunate and sorafenib.

\footnotetext{
${ }^{1}$ Department of Pharmacology, School of Pharmacy, Qingdao University, Qingdao 266021, China

Correspondence: Jian-jun Gao (gaojj@qdu.edu.cn)

These authors contributed equally: Xu Yao, Chen-ru Zhao
}

Received: 29 September 2019 Accepted: 15 March 2020

Published online: 16 April 2020 


\section{MATERIALS AND METHODS}

Agents

Sorafenib and artesunate were obtained from Aladdin (Shanghai, China) and AK Scientific (Union City, CA, USA), respectively. For in vitro studies, sorafenib and artesunate were first dissolved in dimethyl sulfoxide (DMSO; Sigma, St. Louis, MO, USA) and then diluted with culture medium. For in vivo studies, sorafenib was formulated as previously described [21] by dissolving in Cremophor EL/ethanol (50:50) and further diluting with water to the final dosing solution $(1 \mathrm{mg} / \mathrm{mL})$. Artesunate was first dissolved in sodium bicarbonate injection and then diluted with a glucose injection to the final dosing solution $(10 \mathrm{mg} / \mathrm{mL})$. The working solutions of compounds contained $<0.3 \%$ DMSO in their final concentration. Propidium iodide (PI), Hoechst 33258, and CellEvent ${ }^{\mathrm{TM}}$ caspase-3/7 green detection reagent were obtained from Thermo Fisher (Waltham, MA, USA). $N$-acetyl-L-cysteine (NAC) and $2^{\prime}, 7^{\prime}$-dichlorofluorescein diacetate (DCF-DA) were purchased from Sigma. Primary antibodies against $\mathrm{Bax}$ and $\mathrm{BCl}-2$ were purchased from Proteintech (Chicago, IL, USA). p-STAT3, p-ERK1/2, and GAPDH primary antibodies were from Abcam (Cambridge, UK). Appropriate goat anti-mouse and goat anti-rabbit secondary antibodies were purchased from Abcam.

Cell lines and culture

HCC cell lines, including PLC/PRF/5, HuH7, HepG2, Hep3B, and HCCLM3, were obtained from the National Infrastructure of Cell Line Resource (Beijing, China). HuH7 and HCCLM3 cells were cultured in Dulbecco's modified Eagle's medium (DMEM, Gibco, Grand Island, NY, USA), and Hep3B cells were cultured in modified Eagle's medium (MEM, Gibco) supplemented with $10 \%$ fetal bovine serum. HepG2 and PLC/PRF/5 cells were cultured in DMEM and MEM, respectively, supplemented with $10 \%$ fetal bovine serum, $1 \%$ glutamine (Gibco), and $1 \%$ nonessential amino acids (Gibco).

Cell proliferation assay

HCC cells $\left(5 \times 10^{3}\right.$ per well) seeded in 96-well plates were exposed to the indicated drugs for $72 \mathrm{~h}$. Then, the medium was removed, and the wells were washed with phosphate-buffered saline (PBS). Fifteen microliters of 3-(4,5-dimethylthiazol-2-yl)2,5-diphenyltetrazolium bromide (MTT) was added to each well at a working concentration of $5 \mathrm{mg} / \mathrm{mL}$. After incubation for $4 \mathrm{~h}$, the medium was removed, and $150 \mu \mathrm{L}$ of DMSO was added into each well to dissolve the formazan crystals. Light absorbance was measured at $490 \mathrm{~nm}$ on a microplate reader (Tecon, Switzerland).

\section{Cell apoptosis assay}

HCC cell apoptosis was analyzed using Hoechst 33258 (a bluefluorescent dye permeable to both living and dead cells), propidium iodide ( $\mathrm{PI}$, a red fluorescent dye only permeable to dead cells), and CellEvent ${ }^{\mathrm{TM}}$ caspase-3/7 green detection reagent (a green fluorescent dye that indicates apoptotic cells). HCC cells seeded in 96-well plates were incubated with the indicated drugs for $24 \mathrm{~h}$, washed with PBS and stained with $\mathrm{PI}$, caspase-3/7 green detection reagent, and Hoechst 33258. The simultaneous use of these three dyes allows for normal, apoptotic, and dead cells to be distinguished with fluorescence microscopy. We also performed Annexin V-fluorescein isothiocyanate (FITC)/PI staining assays using a cell apoptosis detection kit (BD Biosciences). Apoptotic cells were estimated in a flow cytometer (BD Biosciences).

ROS detection

ROS generation from living cells was measured using the oxidation-sensitive probe $2^{\prime}, 7^{\prime}$-dichlorodihydrofluorescein diacetate (DCF-DA, Sigma), which is cleaved by nonspecific esterases that generate DCF with obvious fluorescence via the oxidation reaction of ROS. Hep3B and $\mathrm{HuH7}$ cells were cultured with artesunate for $2 \mathrm{~h}$ before staining with $20 \mu \mathrm{M}$ DCF-DA in PBS for $30 \mathrm{~min}$ in the dark. Then, the cells were harvested and washed twice with PBS, and the oxidation-induced increase in DCF fluorescence (excitation at $488 \mathrm{~nm}$ and emission at $525 \mathrm{~nm}$ ) was subsequently measured.

\section{Western blot analysis}

HCC cells were lysed using RIPA lysis buffer (Thermo Fisher). The protein concentration was determined by a BCA Protein Assay Reagent Kit (Thermo Fisher). Cell lysates were separated by $10 \%$ SDS-PAGE and electrotransferred onto polyvinylidene fluoride (PVDF) membranes (Millipore). The membranes were blocked with $5 \%$ milk and incubated with antibodies against p-ERK1/2 (1:1000, ab33258, Abcam), p-STAT3 (1:1000, ab76315, Abcam), Bax (1:1000, 50599-2-lg, Proteintech), Bcl-2 (1:1000, 12789-1-AP, Proteintech), PARP1 (1:10,000, 66520-1-lg, Proteintech) and GAPDH (1:10,000, ab181602, Abcam) overnight at $4{ }^{\circ} \mathrm{C}$. The blots were extensively washed with Tris-buffered saline with Tween-20 (TBST) buffer and incubated with horseradish peroxidase-conjugated secondary antibodies. The signal was visualized using an enhanced chemiluminescence reagent (Thermo Fisher) and detected by an ECL Western blotting detection system (Bio-Rad).

In vivo study

The in vivo efficacy of combining artesunate and sorafenib was assessed in a Hep3B xenograft mouse model. Athymic female mice (BALB/c nude), 4-6 weeks of age, were purchased from Beijing Vital River Laboratory Animal Technology Co., Ltd. (Beijing, China). The animals were housed under pathogen-free conditions. The research protocol was in accordance with the institutional guidelines of the Animal Care and Use Committee. Tumors were generated by harvesting Hep3B cells from mid-log phase cultures using trypsin-EDTA (Thermo Fisher). The cells were then pelleted and resuspended in PBS to a final cell count of $5 \times 10^{7} / \mathrm{mL}$. A volume of $0.2 \mathrm{~mL}$ of the cell suspension was injected s.c. in the right flank of each mouse. After 14 days, when the tumor volume had reached $\sim 100 \mathrm{~mm}^{3}$, all the mice were divided into 4 groups $(n=7)$. Mice were administered vehicle, sorafenib (i.g., $10 \mathrm{mg} / \mathrm{kg}$, qd), artesunate (i.p., $100 \mathrm{mg} / \mathrm{kg}$, tid), or both drugs for 21 days. The tumor was resected and weighed at the end of the experiment.

\section{Statistical analysis}

Data are described as the mean \pm SD and were analyzed by oneway analysis of variance (ANOVA) followed by Dunnett's multiple range tests. The limit of statistical significance was $P<0.05$. Statistical analysis was performed with SPSS/Win 16.0 software (SPSS, Chicago, IL, USA).

\section{RESULTS}

Anti-HCC activity of artesunate or sorafenib

We first examined the effect of artesunate or sorafenib alone on cell viability using an MTT assay. Five HCC cell lines, HepG2, PLC/ $\mathrm{PRF} / 5, \mathrm{HCCLM} 3$, Hep3B, and HuH7, were exposed to incremental concentrations of sorafenib $(0.1,0.3,1,3,10,30$, and $100 \mu \mathrm{M})$ or artesunate $(1,3,10,30,100$, and $300 \mu M)$ for $72 \mathrm{~h}$. Sorafenib suppressed the growth of all five HCC cell lines in a dosedependent manner, with $\mathrm{IC}_{50}$ values of $6.1 \mu \mathrm{M}$ (HepG2), $7.4 \mu \mathrm{M}$ (PLC/PRF/5), 14.7 $\mu \mathrm{M}$ (HCCLM3), 3.6 $\mu \mathrm{M}$ (Hep3B), and $2.6 \mu \mathrm{M}$ (HuH7) (Fig. 1a, left panel). Compared with sorafenib, artesunate exhibited a relatively lower inhibitory activity against the proliferation of HCC cells, with $\mathrm{IC}_{50}$ values of $37.4 \mu \mathrm{M}$ (HepG2), $121.2 \mu \mathrm{M}$ (PLC/PRF/5), 44.2 $\mu \mathrm{M}$ (HCCLM3), $41.6 \mu \mathrm{M}$ (Hep3B), and $46.4 \mu \mathrm{M}$ (HuH7) (Fig. 1a, right panel).

As promoting the production of ROS is related to the antitumor activity of artesunate, we measured ROS levels in HuH7 and Hep3B cells before and after treatment with 

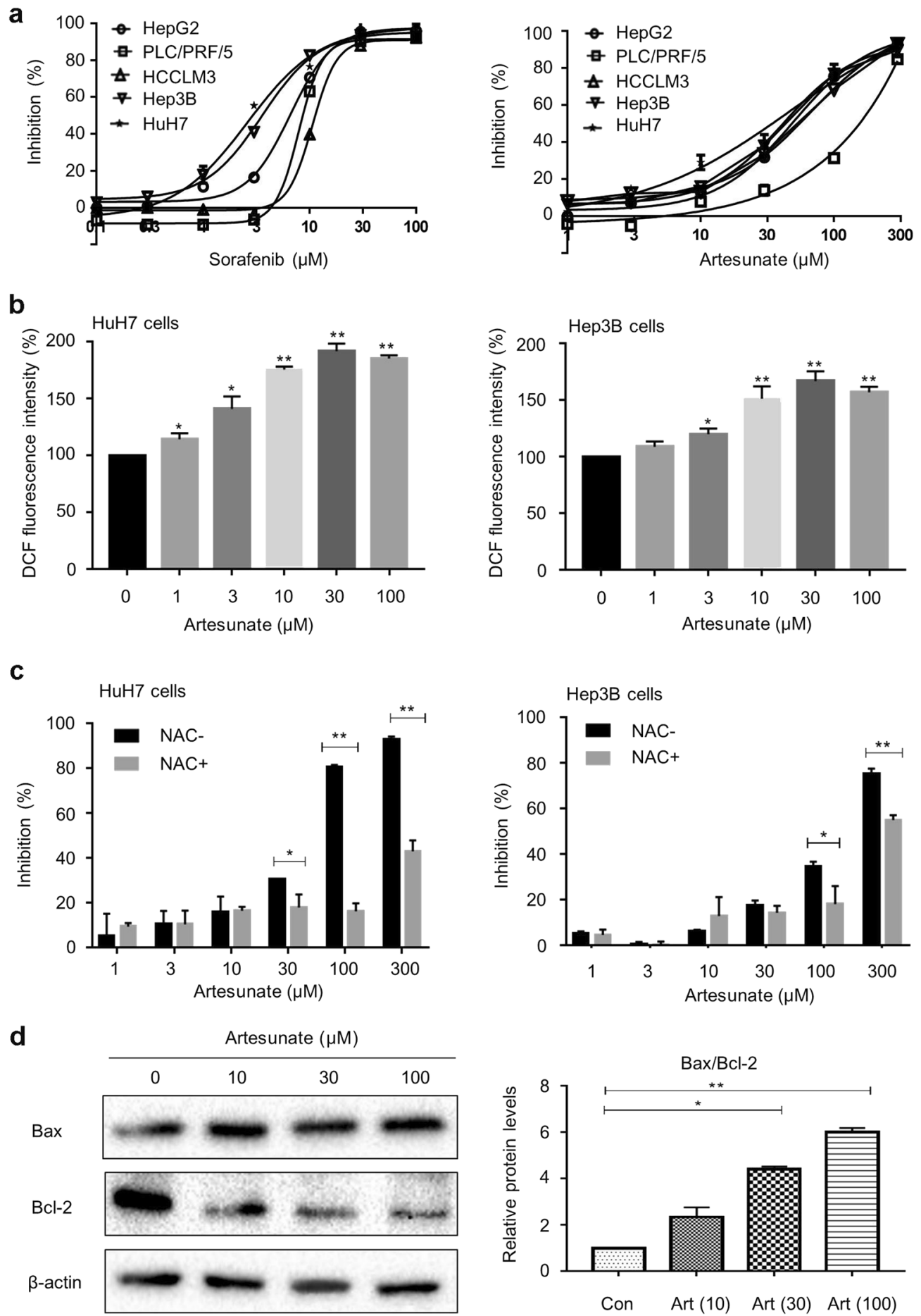

Fig. 1 Anticancer activity of sorafenib or artesunate alone on HCC cells. a HCC cells were incubated with sorafenib or artesunate for $72 \mathrm{~h}$ and then subjected to MTT assay. b ROS levels were detected in HuH7 and Hep3B cells before and after artesunate treatment using fluorometric analysis. c HuH7 and Hep3B cells were treated with artesunate alone or plus $10 \mathrm{mM} \mathrm{N}$-acetyl-L-cysteine (NAC) for $72 \mathrm{~h}$ and then subjected to MTT assay. $\mathbf{d ~ B a x}$ and $\mathrm{BCl}-2$ expression was measured using western blot analysis in HuH7 cells treated with artesunate for $24 \mathrm{~h}$. Con, control; Art, artesunate. ${ }^{*} P<0.05,{ }^{* *} P<0.01$

artesunate. Artesunate treatment dose-dependently increased the ROS level in both HuH7 and Hep3B cells (Fig. 1b). We further confirmed the effect of artesunate on ROS using a membranepenetrating antioxidant, $N$-acetyl-L-cysteine (NAC), which can reduce the intracellular ROS level. As shown in Fig. 1C, NAC significantly ameliorated the inhibitory effect of artesunate on the growth of both $\mathrm{HuH7}$ and Hep3B cells, suggesting that triggering oxidative stress, at least partially, accounts for the anti-HCC activity of artesunate.

It is known that oxidative stress activates proapoptotic factors such as Bax and inactivates antiapoptotic factors such as Bcl-2, leading to cell apoptosis through the mitochondrial pathway. We further examined the effect of artesunate on the expression of these two apoptosis-related proteins using Western blot assays. 

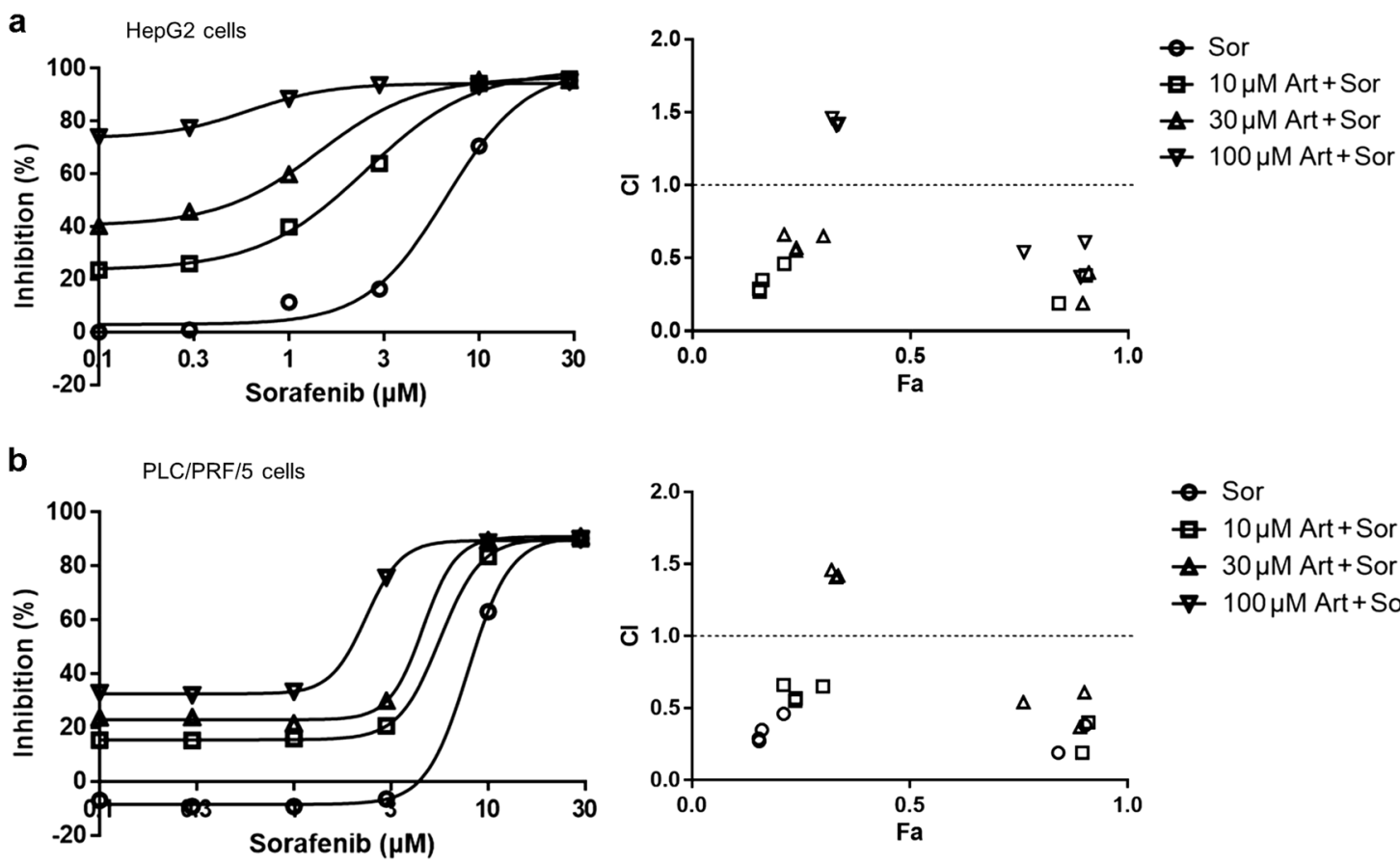

$$
\begin{aligned}
& \text { - Sor } \\
& \text { — } 10 \mu \mathrm{M} \text { Art+Sor } \\
& \text { — } 30 \mu \mathrm{M} \text { Art+Sor } \\
& \text { ₹ } 100 \mu \mathrm{M} \text { Art+Sor }
\end{aligned}
$$
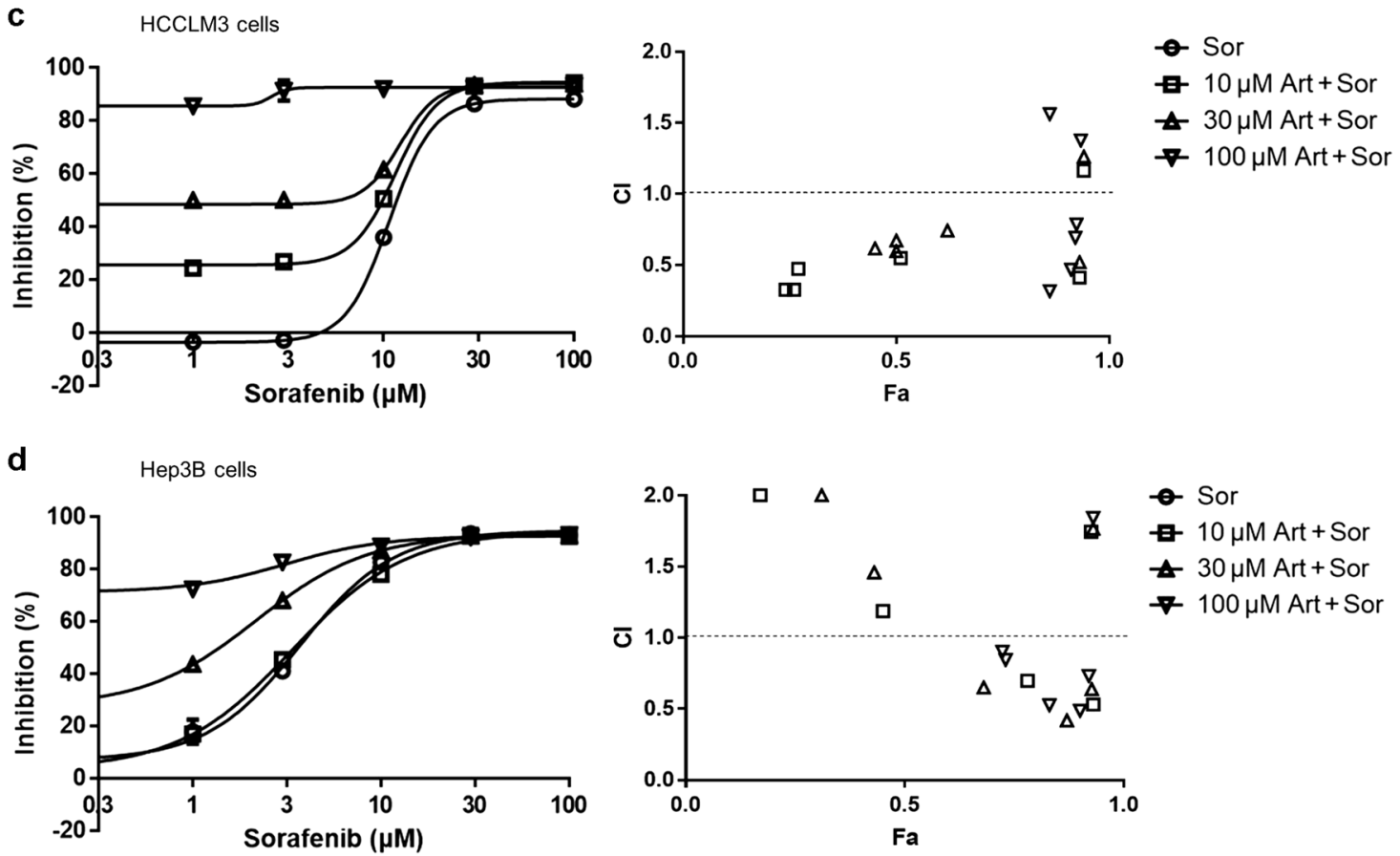

Fig. 2 Artesunate dose-dependently enhances the effect of sorafenib on the suppression of HCC cell proliferation. HepG2 (a), PLC/PRF/5 (b), HCCLM3 (c), and Hep3B (d) cells were exposed to a series of concentrations of sorafenib in the presence or absence of artesunate for $72 \mathrm{~h}$ and then subjected to MTT assay. Cell inhibition rates were plotted against drug concentrations (left panel). Combination indexes (Cls) of sorafenib plus artesunate were calculated and plotted against growth inhibition rates (right panel). Sor, sorafenib; Art, artesunate

As shown in Fig. 1d, artesunate indeed increased the expression of Bax and reduced the expression of $\mathrm{BCl}-2$ in $\mathrm{HuH} 7$ cells, suggesting that artesunate is capable of inducing cell apoptosis by activating the mitochondrial pathway.

Combined sorafenib and artesunate treatment synergistically inhibits HCC cell proliferation

To investigate the anticancer activity of combined sorafenib and artesunate, four HCC cell lines, HepG2, PLC/PRF/5, HCCLM3, and Hep3B, were first treated with incremental levels of sorafenib in the presence or absence of artesunate for $72 \mathrm{~h}$, and cell growth was examined using the MTT assay. Artesunate enhanced the inhibitory effect of sorafenib on the growth of four cell lines in a dose-dependent manner (Fig. 2). Sorafenib ranging from 0 to $30 \mu \mathrm{M}$ resulted in a leftward shift of cell growth inhibition in the presence of 10,30 , or $100 \mu \mathrm{M}$ artesunate compared with sorafenib alone. We also examined the antiproliferation effects of artesunate in the presence or absence of sorafenib. Artesunate at the concentration range of $0-300 \mu \mathrm{M}$ resulted in a leftward shift in cell growth inhibition in four HCC cell lines in the presence of $2.5,5$, or 
a

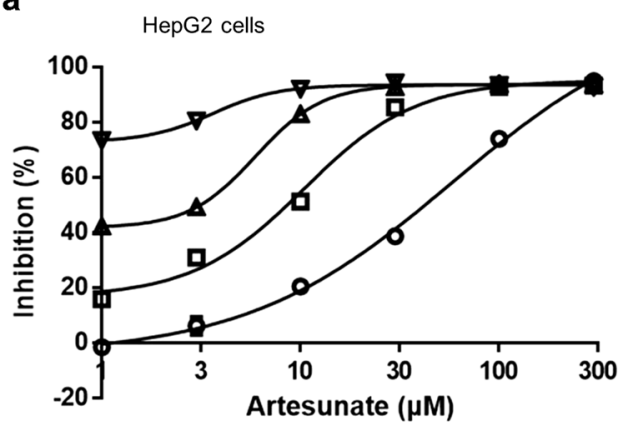

b

b PLC/PRF/5 cells

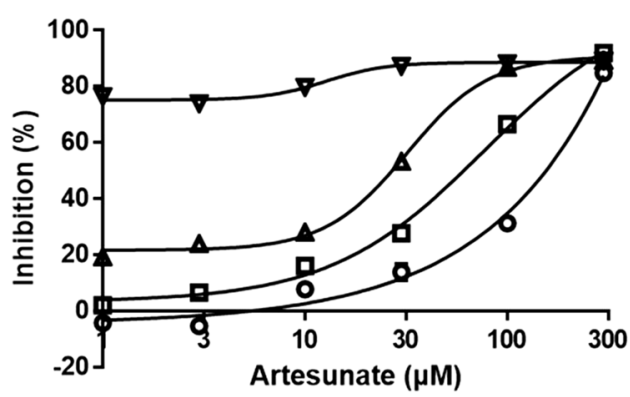

C

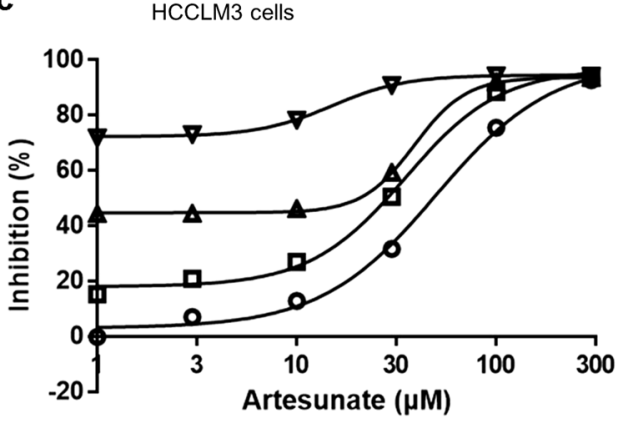

d

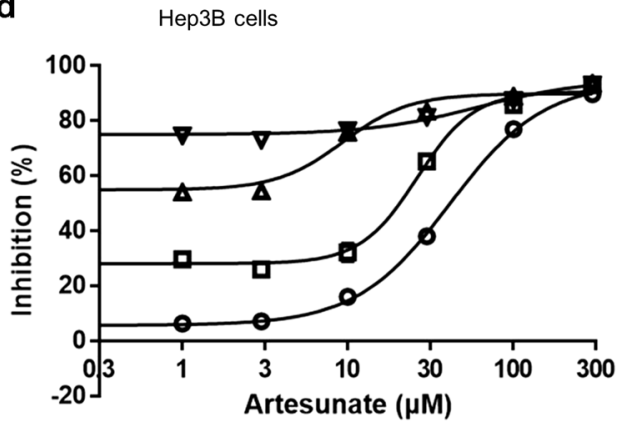

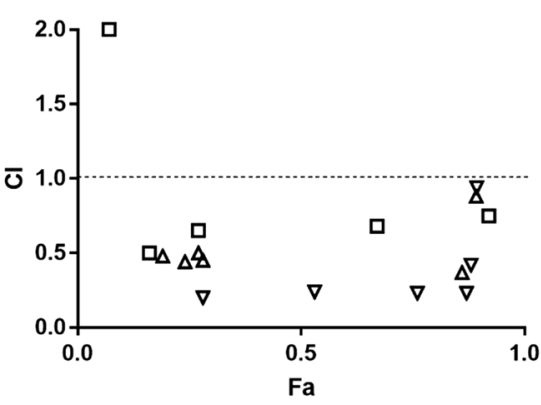

- Art

— $2.5 \mu \mathrm{M}$ Sor+Art

$\triangle 5 \mu \mathrm{M}$ Sor + Art

$\rightarrow 10 \mu \mathrm{M}$ Sor + Art
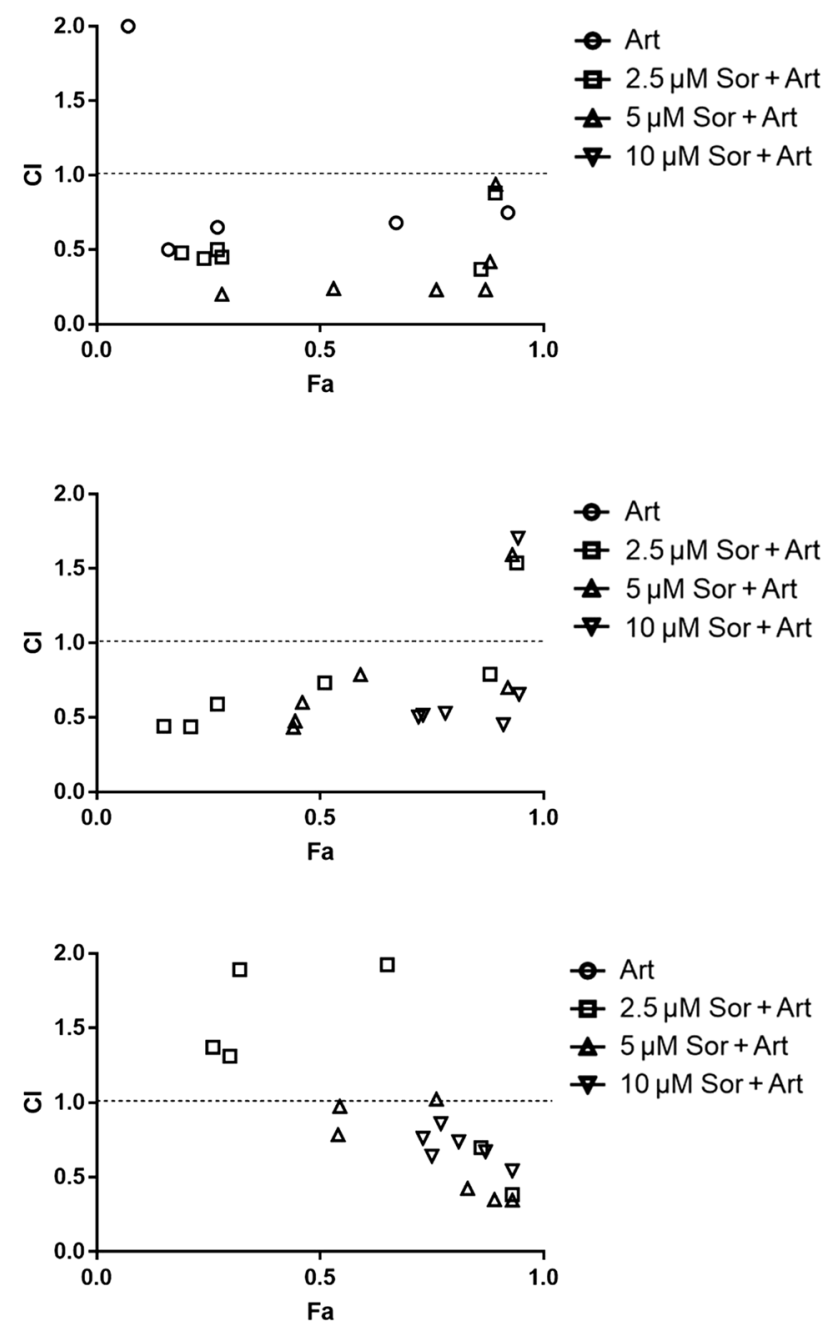

Fig. 3 Sorafenib dose-dependently enhances the effect of artesunate on the suppression of HCC cell proliferation. HepG2 (a), PLC/PRF/5 (b), HCCLM3 (c), and Hep3B (d) cells were exposed to a series of concentrations of artesunate in the presence or absence of sorafenib for $72 \mathrm{~h}$ and then subjected to MTT assay. Cell inhibition rates were plotted with drug concentrations (left panel). Combination indexes (Cls) of sorafenib plus artesunate were calculated and plotted against cell inhibition rates (right panel). Sor, sorafenib; Art, artesunate

$10 \mu \mathrm{M}$ sorafenib compared with artesunate alone (Fig. 3), indicating that sorafenib increased the inhibitory effect of artesunate on the growth of HCC cells in a dose-dependent manner.

The combination index $(\mathrm{Cl})$ values of sorafenib plus artesunate were calculated using compuSyn software, and drug interaction types were ascertained according to the Chou-Talalay method. The results demonstrated that combined sorafenib and artesunate treatment produced synergistic effects at nearly all tested combinations except for those extreme cases in which very low or high concentrations of sorafenib or artesunate were used (Figs. 2, 3, right panels). The synergistic effect between sorafenib and artesunate was observed in all four tested HCC cell lines, indicating that this was not a cell line-specific effect.

Combined sorafenib and artesunate treatment enhances apoptotic cell death

We noticed that artesunate alone increased the proportion of Bax and $\mathrm{BCl}-2$ expression in $\mathrm{HCC}$ cells, suggesting that artesunate 
might be capable of inducing HCC cell apoptosis. We analyzed the cell apoptosis induced by combined sorafenib and artesunate treatment in HCC cell lines. HCC cells were exposed to sorafenib $(10 \mu \mathrm{M})$ in the presence or absence of different concentrations of artesunate $(10,30$, or $100 \mu \mathrm{M})$ for $24 \mathrm{~h}$ and subsequently stained with Hoechst 33258 and PI. As shown in Fig. 4a, c, sorafenib $(10 \mu \mathrm{M})$ alone increased the proportion of PI-stained cells from $2.1 \%$ (vehicle) to $33.3 \%$ in PLC/PRF/5 cells. Artesunate alone at 10 , 30 , or $100 \mu \mathrm{M}$ only increased the proportion of PI-stained cells by $0.6 \%, 0.8 \%$, and $2.7 \%$, respectively. Cotreatment of PLC/PRF/5 cells with sorafenib $(10 \mu \mathrm{M})$ and artesunate at 30 or $100 \mu \mathrm{M}$ significantly increased cell death, as measured by increases of $54 \%$ and $80.5 \%$, respectively (Fig. $4 \mathrm{a}, \mathrm{c}$ ). This enhanced cell death induced by the combination was further observed in $\mathrm{HuH} 7$ cells. Sorafenib or artesunate alone did not induce cell death (Fig. 4b, d). In contrast, combining sorafenib $(10 \mu \mathrm{M})$ and artesunate $(10,30$, or $100 \mu \mathrm{M})$ resulted in a dose-dependent increase in cell death in HuH7 cells (Fig. 4b, d). Using Annexin/PI staining and flow cytometry, we confirmed that the combination of sorafenib and artesunate enhanced cell apoptosis in $\mathrm{HuH} 7$ and Hep3B cells (Fig. 4e, f).

Active caspase-3/7 are well-known central regulators and hallmarks of cell apoptosis. We employed a substrate reagent of active caspase-3/7, which emits green fluorescence after cleavage, to determine cell apoptosis. The proportions of active caspase-3/7positive PLC/PRF/5 cells after exposure to sorafenib $(10 \mu \mathrm{M})$ plus artesunate $(30$ or $100 \mu \mathrm{M})$ were obviously increased by $62 \%$ and $75.6 \%$, respectively, compared with an increase of only $33.3 \%$ after treatment with sorafenib alone (Fig. 5a). Caspase-3/7-stained HuH7 and Hep3B cells were not obviously detected after exposure to sorafenib or artesunate alone but were significantly increased after exposure to the combination (Fig. 5b, c). We checked the level of poly (ADP-ribose) polymerase 1 (PARP1), a substrate of activated caspase- $3 / 7$, in HuH7 cells exposed to sorafenib, artesunate, and the drug combination. The results demonstrated that the levels of cleaved PARP1 were obviously increased after treatment with both sorafenib and artesunate compared with the individual drug (Fig. 5d). Furthermore, we examined the effect of sorafenib and artesunate combination treatment on the expression of intrinsic apoptotic markers Bax and $\mathrm{BCl}-2$ using Western blot analysis. The results showed that combined sorafenib and artesunate treatment increased Bax expression and decreased $\mathrm{Bcl}-2$ expression in $\mathrm{HuH} 7$ cells (Fig. 5d). The combined treatment more efficiently increased the $\mathrm{Bax} / \mathrm{BCl}-2$ ratio than did either drug used alone. The above results indicate that combined sorafenib and artesunate treatment is obviously superior to the individual drugs in promoting cell apoptosis via the mitochondria-dependent pathway.

Increased anticancer sensitivity of sorafenib through the enhancement of $p$-ERK and p-STAT3 signaling by artesunate The efficacy of molecularly targeted drugs is usually dependent on the nature and abundance of targeted molecules [22]. Sorafenib suppresses the growth of HCC cells by targeting RAF/MEK/ERK and STAT3 signaling $[23,24]$. A phase II study of sorafenib in patients with advanced HCC showed that tumors containing higher levels of $p$-ERK are more sensitive to sorafenib. To investigate the mechanisms accounting for the synergism of combined sorafenib and artesunate, alterations in ERK and STAT3 signaling were examined in HCC cells exposed to sorafenib, artesunate, or their combination. As expected, sorafenib decreased p-ERK and p-STAT3 levels in a dose-dependent manner in HuH7 cells (Fig. 6a). Intriguingly, we found that artesunate dose-dependently upregulated both p-ERK and p-STAT3 levels in the cells (Fig. 6a). We next examined the alterations in p-ERK and p-STAT3 after exposure to combined sorafenib and artesunate. The results demonstrated that artesunate alone significantly increased the levels of p-ERK and p-STAT3, but the effect was abrogated by sorafenib (Fig. 6b). We further confirmed that artesunate is capable of increasing
p-ERK and p-STAT3 levels in Hep3B cells, which was significantly suppressed by sorafenib when used in combination (Fig. 6c, d). It is well documented that the overexpression of activated ERK and STAT3 in HCC cells promotes tumor growth and survival and prevents cell apoptosis $[25,26]$. We speculated that the excessive ROS accumulation induced by artesunate promoted cell apoptosis, while the upregulation of p-ERK and p-STAT3 signaling by artesunate facilitated cell survival to maintain self-balance in HCC cells. To verify this hypothesis, we determined the anticancer effect of artesunate in combination with a selective inhibitor of ERK phosphorylation (PD0325901) or a specific STAT3 inhibitor (Stattic). The results of the MTT assay demonstrated that combining artesunate with PD0325901 or Stattic produced synergism in suppressing the growth of $\mathrm{HuH7}$ and Hep3B cells (Fig. 7a, b). In addition, combined artesunate and PD0325901 or Stattic was superior to the individual drugs in activating caspase-3/7 and soliciting cell apoptosis (Fig. 7c-f). Western blotting confirmed that PD0325901 and Stattic reduced the levels of $p$-ERK and p-STAT3, which were increased by artesunate treatment (Fig. 7g). These results suggested that the activation of ERK and STAT3 signaling by artesunate probably accounts for the synergistic effect of combined artesunate and sorafenib on the inhibition of HCC cell growth.

Combined sorafenib and artesunate enhances antitumor efficacy in vivo

We assessed the in vivo antitumor efficacy of sorafenib plus artesunate in nude mice bearing Hep3B xenografts. Mice were randomly assigned to four groups: control, sorafenib, artesunate, and combination treatment. Compared with the control condition, the oral administration of sorafenib at $10 \mathrm{mg} / \mathrm{kg}$ qd or i.p. injection of artesunate at $100 \mathrm{mg} / \mathrm{kg}$ tid for 21 consecutive days resulted in significant tumor growth inhibition (TGI) of approximately $32.2 \%$ and $37.1 \%$, respectively (Fig. 8). Combining sorafenib and artesunate resulted in significantly increased tumor growth suppression with a TGI of $\sim 53.9 \%$ compared with sorafenib or artesunate alone (Fig. 8). No significant change in body weight was found in mice that received sorafenib compared with control mice at the end of the experiment. Compared with the control mice, the mice that received artesunate and the drug combination lost $8.7 \%$ and $11.2 \%$ of body weight, respectively. No significant difference in body weight was observed between the two groups.

\section{DISCUSSION}

The goal of this study was to examine the inhibition of HCC cells by the combination of sorafenib and artesunate. We found that the drug combination synergistically suppressed the cell proliferation of four HCC cell lines. This combination treatment also resulted in an increase in the $\mathrm{Bax} / \mathrm{Bcl}-2$ ratio, activated caspase- $3 / 7$ levels, and apoptosis in HCC cells. The anti-HCC effect of artesunate is, at least in part, ascribed to its ability to promote ROS accumulation. We also found that artesunate increased the levels of $p$-ERK and p-STAT3, while sorafenib decreased the levels of $p$-ERK and p-STAT3. When used in combination, sorafenib obviously suppressed the activation of ERK and STAT3 induced by artesunate, which might account for the synergistic anti-HCC activity of combined sorafenib and artesunate.

Previous studies have demonstrated that artemisinin and its analogs selectively cause apoptosis in multiple cancer cell lines $[16,27]$. However, the mechanisms underlying the anticancer activity of artemisinin and its derivatives are incompletely elucidated. One viewpoint is that cancer cells contain significantly more intracellular free iron than normal cells, while artemisinin contains an endoperoxide moiety that can react with iron to form cytotoxic ROS [28]. ROS, including superoxide anion $\left(\mathrm{O}_{2}{ }^{-}\right)$, hydrogen peroxide $\left(\mathrm{H}_{2} \mathrm{O}_{2}\right)$, and hydroxyl radicals $(\mathrm{OH} \cdot)$, have inherent chemical properties that confer reactivity to different 
a

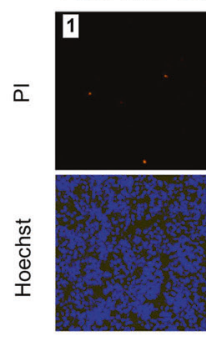

b

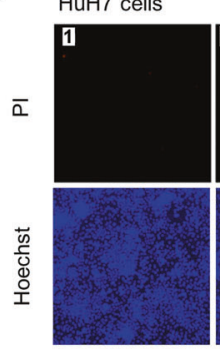

C
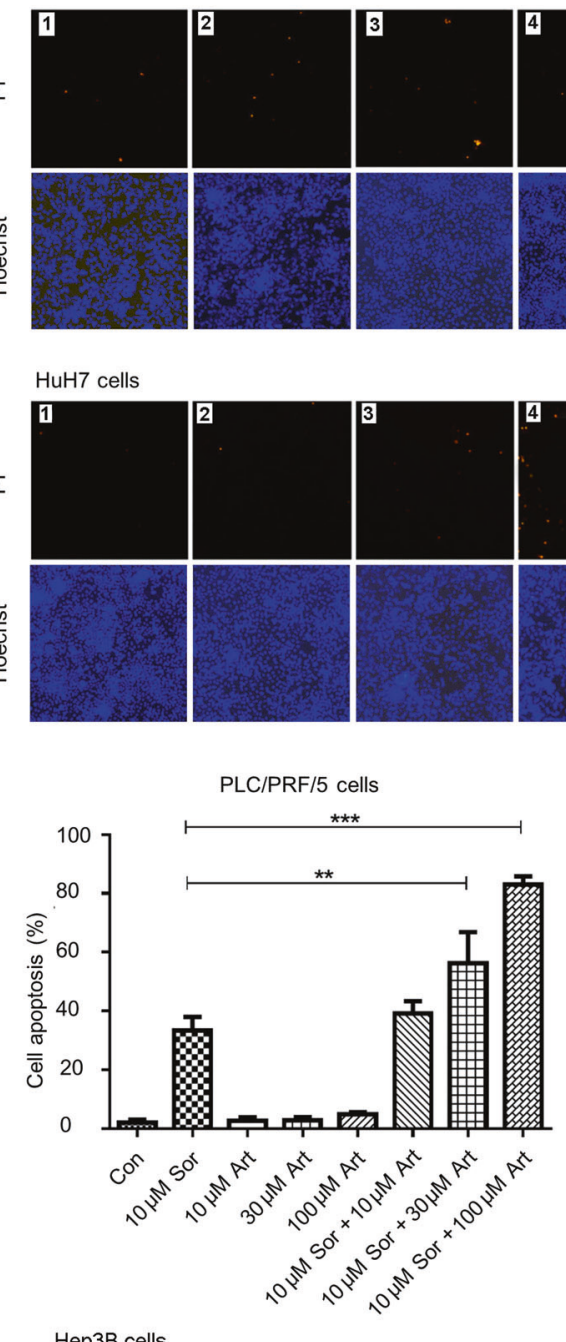

e
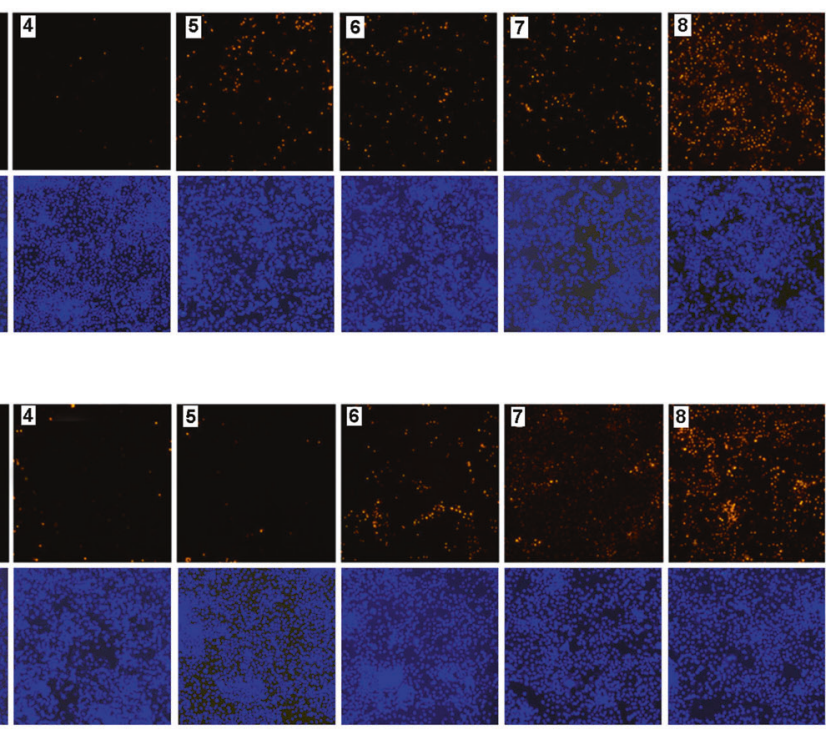

d

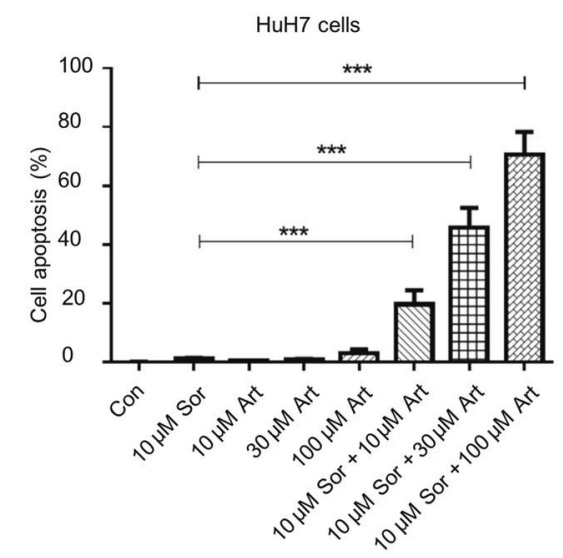

Hep3B cells
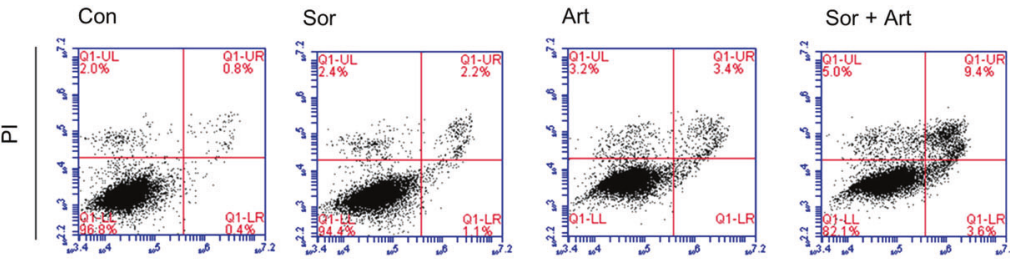

Annxin V

f

HuH7 cells Con

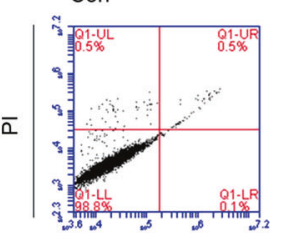

Sor

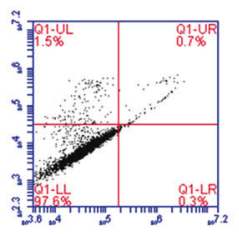

Art

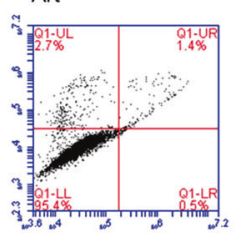

Sor + Art

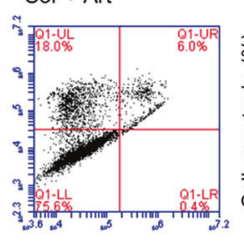

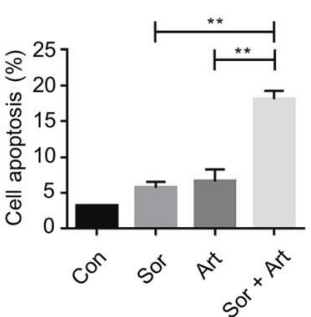

Annxin V

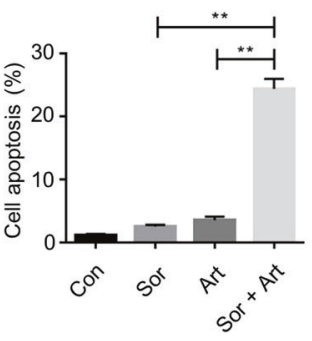

Fig. 4 Combining sorafenib and artesunate enhances cell apoptosis. HCC cells were treated with sorafenib, artesunate, or their combination for $24 \mathrm{~h}$. a-d PLC/PRF/5 and HuH7 cells were stained with PI and Hoechst 33258. 1, Control; $2,10 \mu \mathrm{M}$ artesunate; 3, 30 $\mu \mathrm{M}$ artesunate; 4, $100 \mu \mathrm{M}$ artesunate; $5,10 \mu \mathrm{M}$ sorafenib; $6,10 \mu \mathrm{M}$ artesunate plus $10 \mu \mathrm{M}$ sorafenib; $7,30 \mu \mathrm{M}$ artesunate plus $10 \mu \mathrm{M}$ sorafenib; $8,100 \mu \mathrm{M}$ artesunate plus $10 \mu \mathrm{M}$ sorafenib. The percentage of apoptotic cells was quantified by counting the numbers of PI- and Hoechst 33258-stained cells and calculating the ratio of the two. e, f Hep3B (e) and $\mathrm{HuH7}(\mathbf{f})$ cells were stained with Annexin V/PI, and the cell apoptotic ratio was measured by flow cytometry analysis. Con, control; Sor, sorafenib; Art, artesunate. ${ }^{* *} P<0.01,{ }^{* * *} P<0.001$ 
a PLC/PRF/5 cells
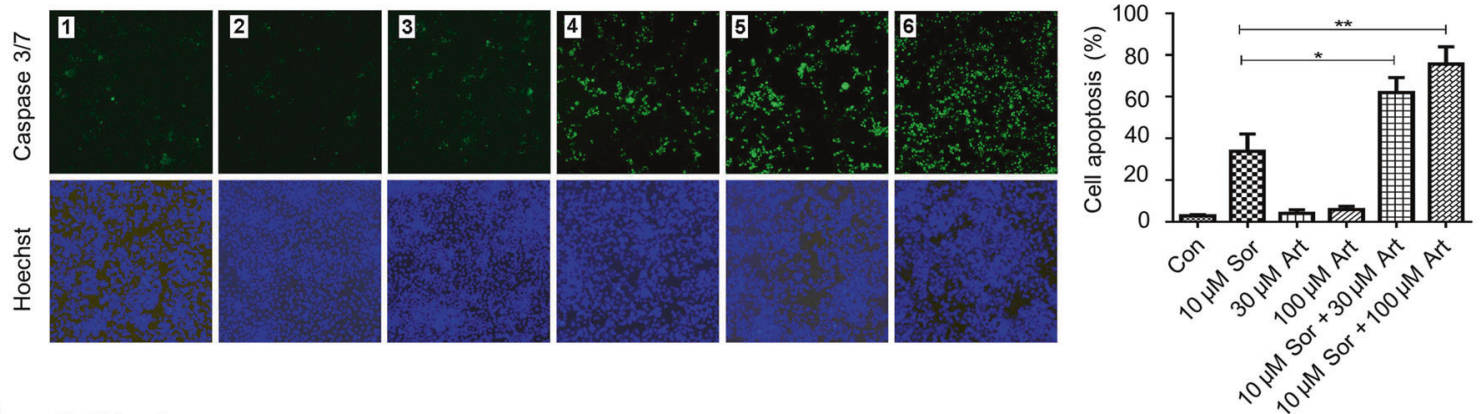

b
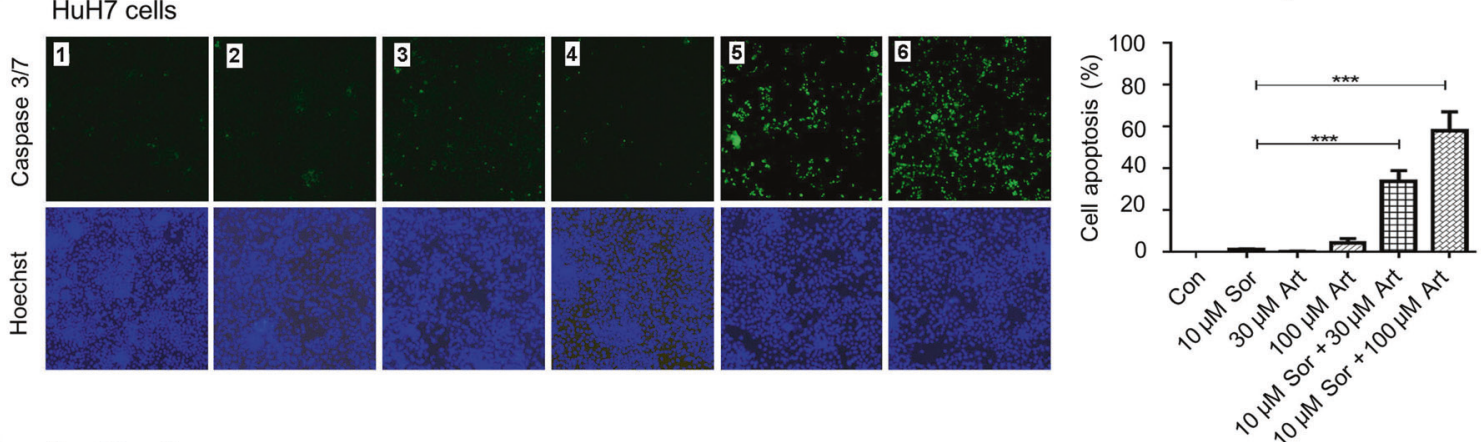

C
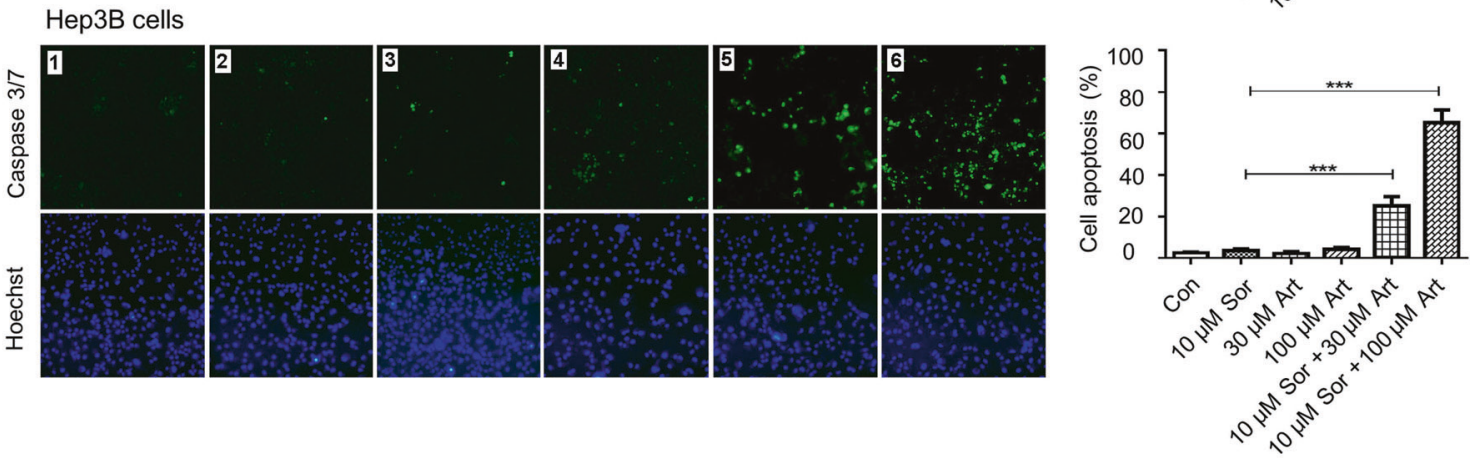

d
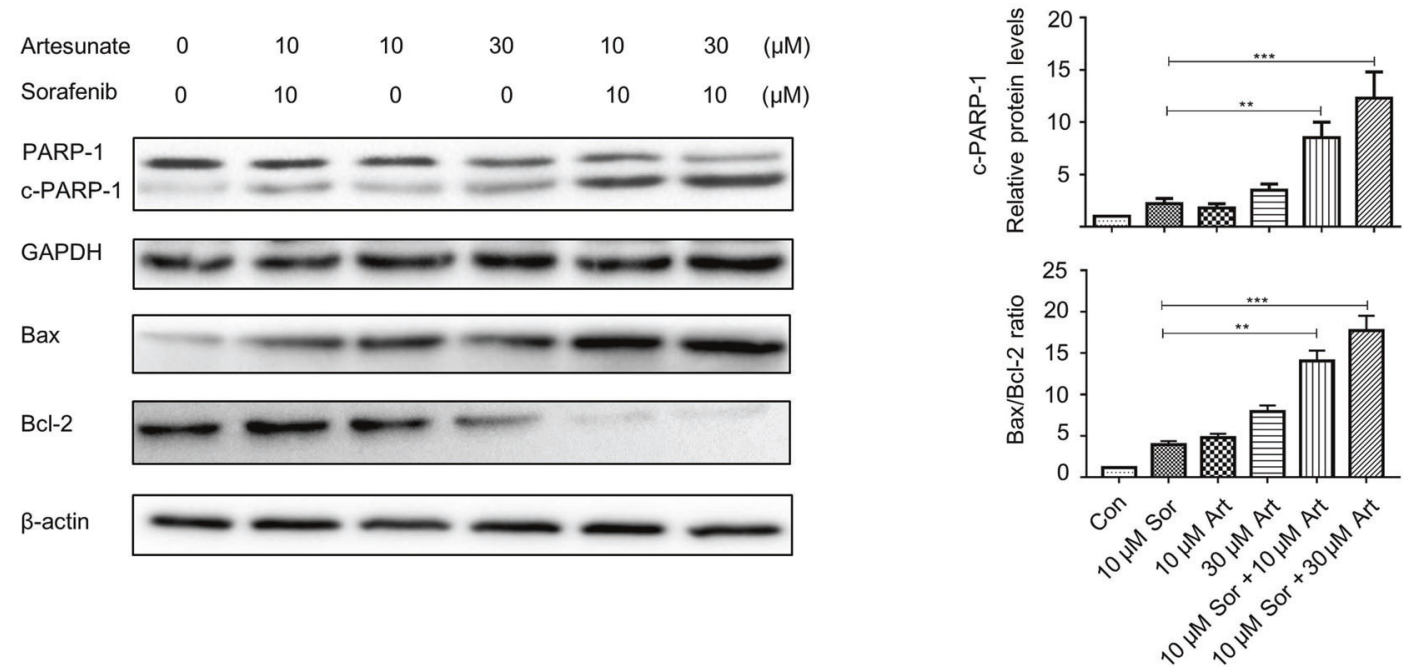

Fig. 5 The combined use of sorafenib and artesunate increases the levels of apoptosis-related proteins. a-c PLC/PRF/5, HuH7, and Hep3B cells were treated with sorafenib, artesunate, or both for $24 \mathrm{~h}$. Cells were stained with active caspase-3/7 detection reagent and Hoechst 33258 . 1 , Control; $2,30 \mu \mathrm{M}$ artesunate; $3,100 \mu \mathrm{M}$ artesunate; $4,10 \mu \mathrm{M}$ sorafenib; $5,30 \mu \mathrm{M}$ artesunate plus $10 \mu \mathrm{M}$ sorafenib; $6,100 \mu \mathrm{M}$ artesunate plus $10 \mu \mathrm{M}$ sorafenib. The percentage of apoptotic cells was quantified by counting the numbers of active caspase-3/7-positive and Hoechst 33258positive cells and calculating the ratio of the two. $\mathbf{d}$ The levels of PARP1, Bax and Bcl-2 were determined by Western blot analysis in HuH7 cells, and their ratios were calculated. Con, control; Sor, sorafenib; Art, artesunate. ${ }^{*} P<0.05$, ${ }^{* *} P<0.01,{ }^{* * *} P<0.001$ 
a
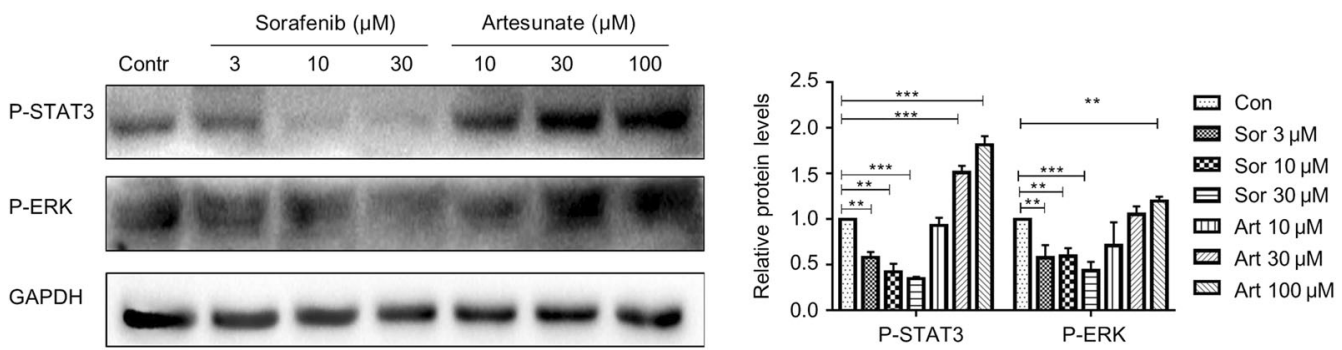

b
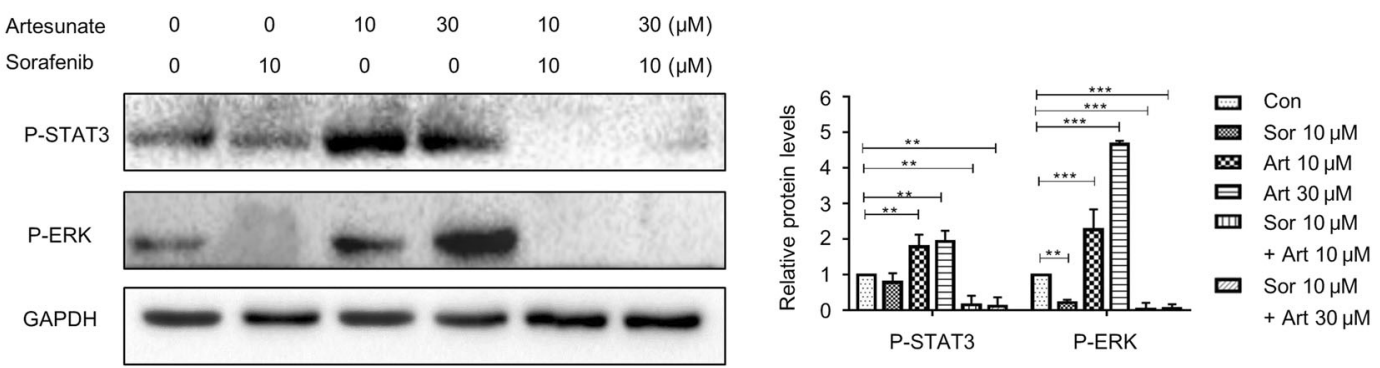

C
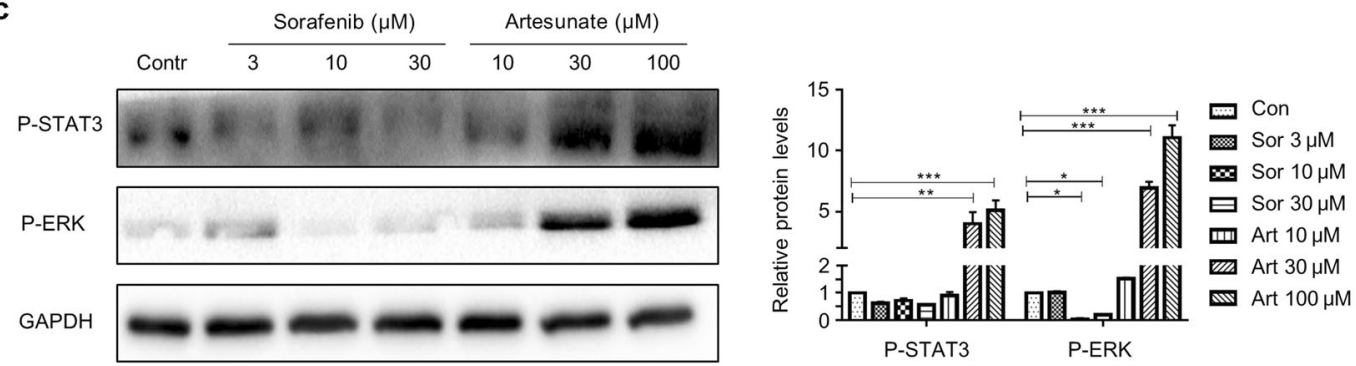

d
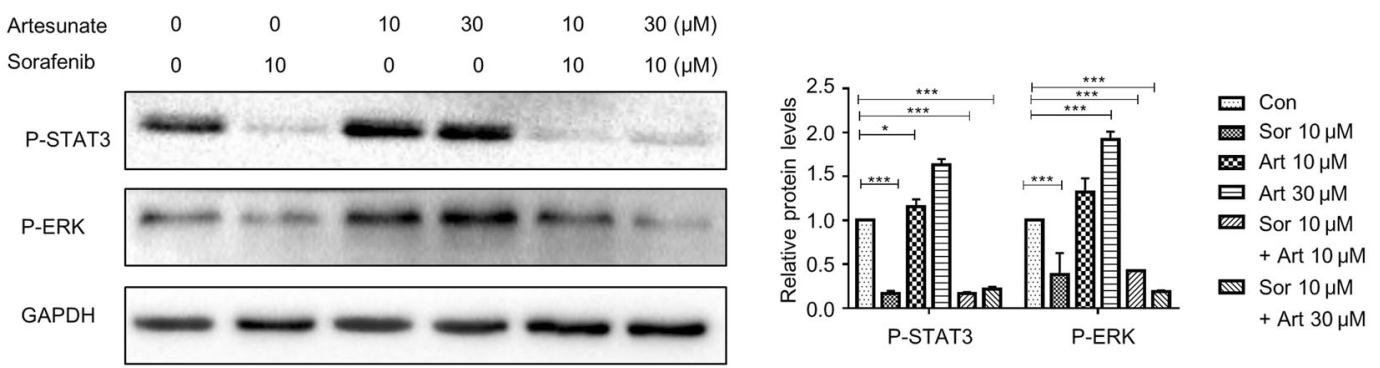

Fig. 6 Sorafenib suppresses the increase in p-STAT3 and p-ERK levels by artesunate. HuH7 and Hep3B cells were exposed to sorafenib, artesunate, or both for $24 \mathrm{~h}$. The expression of p-STAT3 and p-ERK was detected by Western blot analysis. Sorafenib dose-dependently reduced the levels of p-STAT3 and p-ERK, while artesunate dose-dependently increased the levels of p-STAT3 and p-ERK in HuH7 (a) and Hep3B cells (c). When used in combination, sorafenib suppressed the increase in p-STAT3 and p-ERK levels by artesunate in HuH7 (b) and Hep3B (d) cells. Con, control; Sor, sorafenib; Art, artesunate. ${ }^{*} P<0.05,{ }^{*} P<0.01,{ }^{* *} P<0.001$

biological targets [29]. ROS cause toxicity to cancer cells by oxidizing lipids, proteins, and DNA, eventually leading to cell damage or genomic instability [29]. Our results reveal that the antioxidant NAC could effectively reduce the cytotoxic effect of artesunate on HCC cells, suggesting that the induction of ROS contributes to the anti-HCC activity of artesunate.

Why does the drug combination produce synergistic anti-HCC activity? We reason that this synergism might be ascribed to the activation of the ERK and STAT3 signaling pathways by artesunate, which acts as a self-balancing mechanism that facilitates the survival of HCC cells. Coincidentally, a previous study showed that artesunate attenuated the myocardial injury associated with ischemia/reperfusion (I/R) in an animal model via the activation of ERK and STAT3 signaling [30]. The activation of
ERK and STAT3 signaling by artesunate is possibly correlated with its ROS induction [31, 32]. Although historically viewed as purely harmful, multiple lines of evidence suggest that ROS function as important regulators of intracellular signaling pathways involved in cell growth factor stimulation $[29,31]$. The effects of ROS on signal transduction are produced in large part through the covalent modification of specific cysteine residues found within redox-sensitive target proteins such as protein tyrosine phosphatases (PTPs) [33]. PTPs can act as negative regulators of signal transduction pathways by dephosphorylating the receptor tyrosine kinase itself or its downstream target. Thus, the oxidation and inactivation of PTPs by ROS reinforces proliferative signaling pathways. Our results demonstrated that p-ERK and p-STAT3 are upregulated in $\mathrm{HuH7}$ and Hep3B cells after treatment 
a
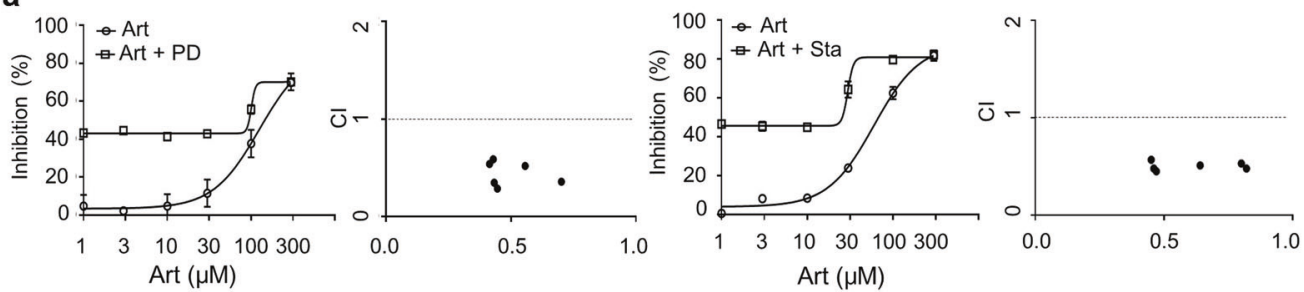

b
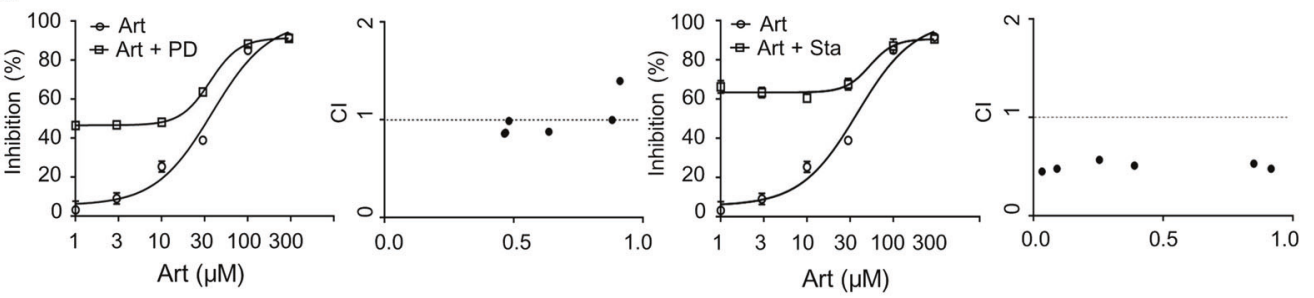

c
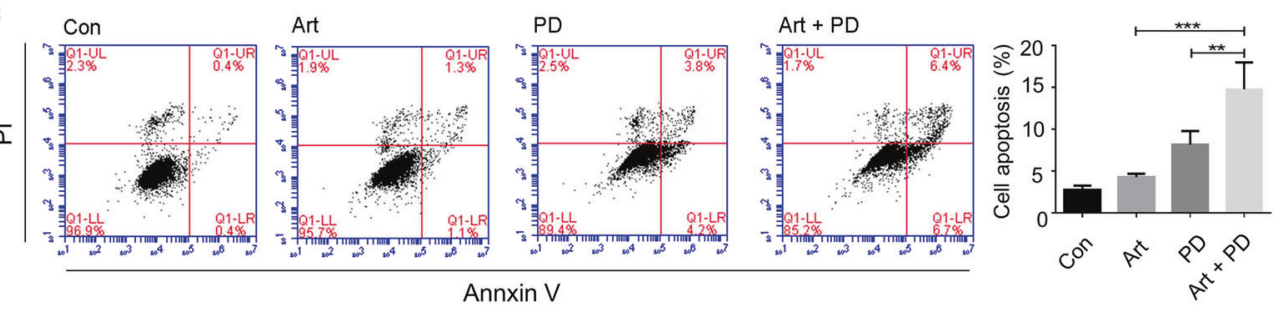

d
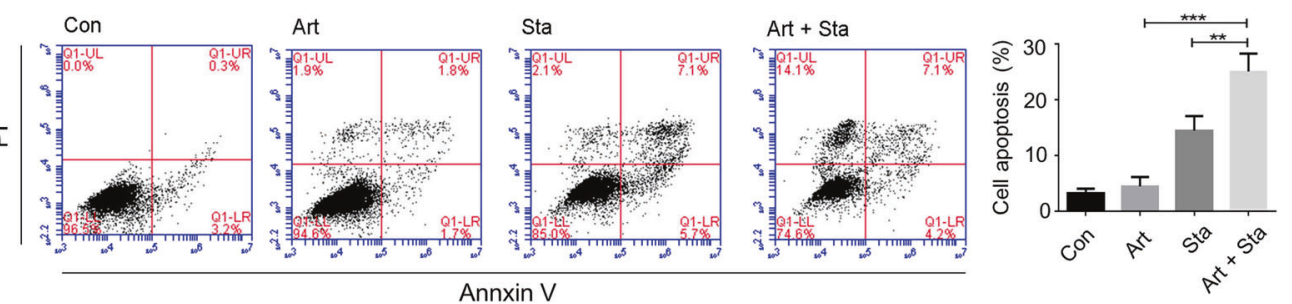

e
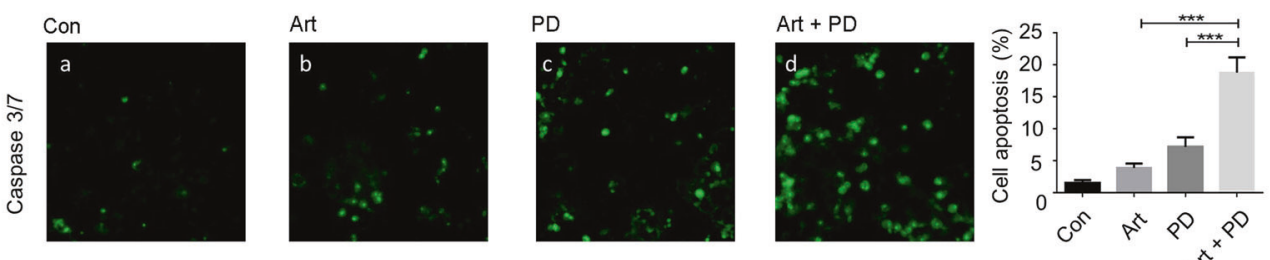

f
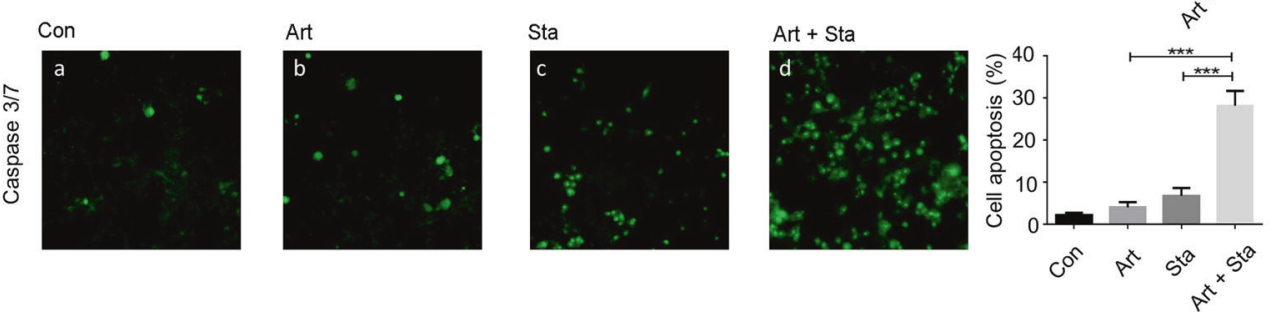

g
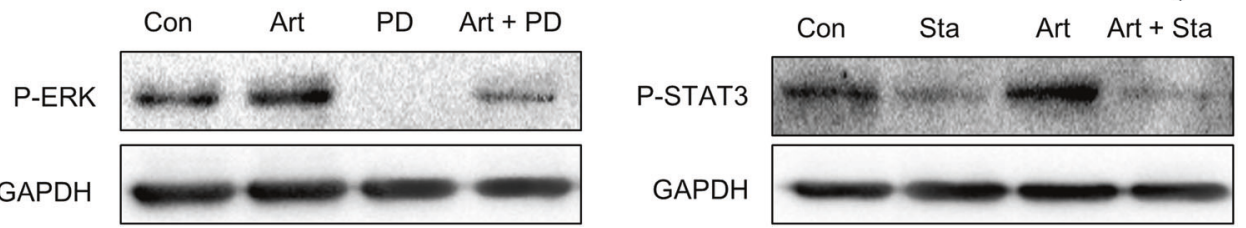

Fig. 7 The combination of artesunate and the ERK-specific inhibitor PD0325901 or the STAT3-specific inhibitor Stattic enhanced anti-HCC activity. a, b Hep3B (a) and HuH7 (b) cells were incubated with incremental concentrations of artesunate in the presence or absence of PD0325901 $(1 \mu \mathrm{M})$ or Stattic $(3 \mu \mathrm{M})$ for $72 \mathrm{~h}$ before being subjected to MTT assay. Cell inhibition rates were plotted against drug concentrations (left panel). Combination indexes (Cls) of artesunate plus PD0325901 or Stattic were calculated and plotted with cell inhibition rates (right panel). c-g HuH7 cells were incubated with artesunate $(30 \mu \mathrm{M})$, PD0325901 $(1 \mu \mathrm{M})$, Stattic $(3 \mu \mathrm{M})$, artesunate plus PD0325901, or artesunate plus Stattic for $24 \mathrm{~h}$. Cells were stained with Annexin V/PI, and the cell apoptosis ratio was measured by flow cytometry analysis (c, d). Cells were stained with active caspase-3/7 detection reagent to examine the levels of activated caspase-3/7 (e, f). Cells were lysed and subjected to Western blotting to determine the levels of p-ERK and p-STAT3 (g). Con, Control; Art, artesunate; PD, PD0325901; Sta, Stattic. ${ }^{* * P} P<0.01,{ }^{* * *} P<0.001$ 
a

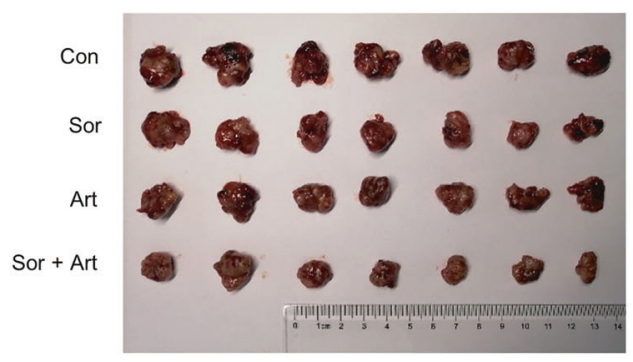

b

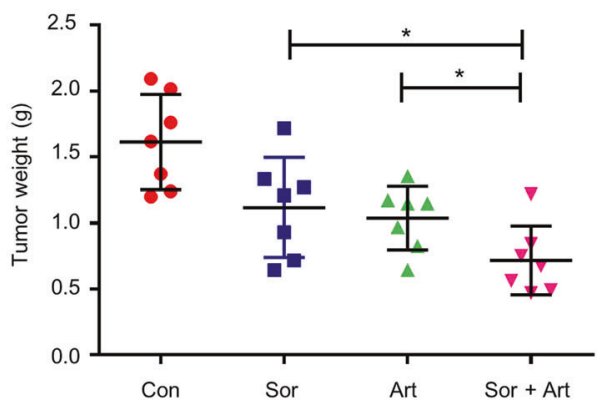

Fig. 8 Enhanced inhibition of xenograft tumor growth in nude mice by the combined use of sorafenib and artesunate. Nude mice with Hep3B xenografts received vehicle, sorafenib (i.g., $10 \mathrm{mg} / \mathrm{kg}$, qd), artesunate (i.p., $100 \mathrm{mg} / \mathrm{kg}$, tid), and the drug combination for $21 \mathrm{days}$. The tumor was resected (a) and weighed (b) at the end of the experiment. Con, control; Sor, sorafenib; Art, artesunate. ${ }^{*} P<0.05$

with artesunate and that either ERK inhibitor PD0325901 or STAT3 inhibitor Stattic could synergistically enhance the activity of artesunate, suggesting that the suppression of ERK and STAT3 signaling by sorafenib contributes to the synergistic activity against HCC when used in combination with artesunate.

In conclusion, we found that the combination of sorafenib and artesunate synergistically suppressed cell proliferation and induced cell apoptosis in HCC cells. ROS accumulation, at least in part, accounts for the anti-HCC activity of artesunate. The activation of ERK and STAT3 by artesunate, which is suppressed by sorafenib, contributes the synergistic anti-HCC activity when two drugs are used in combination. Our findings provide evidence for the potential use of sorafenib plus artemisinin analogs for the new treatment of HCC.

\section{ACKNOWLEDGEMENTS}

This project was supported by grants from the National Natural Science Foundation of China (81503094, 81573410, and 81603071), the China Postdoctoral Science Foundation (2016M600524), and the Qingdao Postdoctoral Applied Research Project (2016072; Jian-jun Gao, Qingdao University).

\section{AUTHOR CONTRIBUTIONS}

JJG conceived and designed the study. $X Y, C R Z$, and HY performed the study. $X Y$ and CRZ collected and analyzed the data. JJG and KWW prepared the article.

\section{ADDITIONAL INFORMATION}

Competing interests: The authors declare no competing interests.

\section{REFERENCES}

1. Kanwal F, Singal AG. Surveillance for HCC: current best practice and future direction. Gastroenterology. 2019;157:54-64.

2. Song $\mathrm{P}$, Cai $\mathrm{Y}$, Tang $\mathrm{H}$, Li C, Huang J. The clinical management of hepatocellular carcinoma worldwide: a concise review and comparison of current guidelines from 2001 to 2017. Biosci Trends. 2017;11:389-98.

3. Sun Z, Zhu Y, Aminbuhe, Fan Q, Peng J, Zhang N. Differential expression of APE1 in hepatocellular carcinoma and the effects on proliferation and apoptosis of cancer cells. Biosci Trends. 2018;12:456-62.

4. Yan X, Qiu Y. Impact of current staging systems on treatment strategy for HBVrelated hepatocellular carcinoma. Cancer Lett. 2016;379:220-4.

5. Gao J, Song P. Combination of triple biomarkers AFP, AFP-L3, and PIVAKII for early detection of hepatocellular carcinoma in China: expectation. Drug Disco Ther. 2017;11:168-9.

6. Dimitroulis D, Damaskos C, Valsami S, Davakis S, Garmpis N, Spartalis E, et al. From diagnosis to treatment of hepatocellular carcinoma: an epidemic problem for both developed and developing world. World J Gastroenterol. 2017;23:5282-94.

7. Qi F, Wang J, Zhao L, Cai P, Tang W, Wang Z. Cinobufacini inhibits epithelialmesenchymal transition of human hepatocellular carcinoma cells through c-Met/ ERK signaling pathway. Biosci Trends. 2018;12:291-7.
8. Cheng AL, Kang YK, Chen Z, Tsao CJ, Qin S, Kim JS, et al. Efficacy and safety of sorafenib in patients in the Asia-Pacific region with advanced hepatocellular carcinoma: a phase III randomised, double-blind, placebo-controlled trial. Lancet Oncol. 2009;10:25-34.

9. Llovet JM, Ricci S, Mazzaferro V, Hilgard P, Gane E, Blanc JF, et al. Sorafenib in advanced hepatocellular carcinoma. N Engl J Med. 2008;359:378-90.

10. Peng Y, Li Q, Zhang J, Shen W, Zhang X, Sun C, et al. Update review of skin adverse events during treatment of lung cancer and colorectal carcinoma with epidermal growth receptor factor inhibitors. Biosci Trends. 2019;12:537-52.

11. Elket N, Kendjo E, Thellier M, Assoumou L, Potard V, Taieb A, et al. Propensity score analysis of artesunate versus quinine for severe imported Plasmodium falciparum malaria in France. Clin Infect Dis. 2020;70:280-7.

12. Krishna S, Ganapathi S, Ster IC, Saeed ME, Cowan M, Finlayson C, et al. A randomised, double blind, placebo-controlled pilot study of oral artesunate therapy for colorectal cancer. EBioMedicine. 2015;2:82-90.

13. Vatsveen TK, Myhre MR, Steen CB, Walchli S, Lingjaerde OC, Bai B, et al. Artesunate shows potent anti-tumor activity in B-cell lymphoma. J Hematol Oncol. 2018;11:23.

14. Sun $X$, Yan P, Zou C, Wong YK, Shu Y, Lee YM, et al. Targeting autophagy enhances the anticancer effect of artemisinin and its derivatives. Med Res Rev. 2019;39:2172-93.

15. llamathi M, Santhosh S, Sivaramakrishnan V. Artesunate as an anti-cancer agent targets Stat-3 and favorably suppresses hepatocellular carcinoma. Curr Top Med Chem. 2016;16:2453-63.

16. Vandewynckel YP, Laukens D, Geerts A, Vanhove C, Descamps B, Colle I, et al. Therapeutic effects of artesunate in hepatocellular carcinoma: repurposing an ancient antimalarial agent. Eur J Gastroenterol Hepatol. 2014;26:861-70.

17. ClinicalTrials.gov. https://www.clinicaltrials.gov/ct2/results?cond=\&term=artesunate \&cntry $=\&$ state $=\&$ city $=\&$ dist $=(2019)$.

18. Morris CA, Duparc S, Borghini-Fuhrer I, Jung D, Shin CS, Fleckenstein L. Review of the clinical pharmacokinetics of artesunate and its active metabolite dihydroartemisinin following intravenous, intramuscular, oral or rectal administration. Malar J. 2011;10:263.

19. Greenshields AL, Fernando W, Hoskin DW. The anti-malarial drug artesunate causes cell cycle arrest and apoptosis of triple-negative MDA-MB-468 and HER2enriched SK-BR-3 breast cancer cells. Exp Mol Pathol. 2019;107:10-22.

20. Zhang SS, Ni YH, Zhao CR, Qiao Z, Yu HX, Wang LY, et al. Capsaicin enhances the antitumor activity of sorafenib in hepatocellular carcinoma cells and mouse xenograft tumors through increased ERK signaling. Acta Pharmacol Sin. 2018;39:438-48.

21. Wilhelm SM, Carter C, Tang L, Wilkie D, McNabola A, Rong H, et al. BAY 43-9006 exhibits broad spectrum oral antitumor activity and targets the RAF/MEK/ERK pathway and receptor tyrosine kinases involved in tumor progression and angiogenesis. Cancer Res. 2004;64:7099-109.

22. Moriguchi M, Umemura A, Itoh Y. Current status and future prospects of chemotherapy for advanced hepatocellular carcinoma. Clin J Gastroenterol. 2016;9: 184-90.

23. Abou-Alfa GK, Schwartz L, Ricci S, Amadori D, Santoro A, Figer A, et al. Phase II study of sorafenib in patients with advanced hepatocellular carcinoma. J Clin Oncol. 2006;24:4293-300.

24. Tai WT, Cheng AL, Shiau CW, Huang HP, Huang JW, Chen PJ, et al. Signal transducer and activator of transcription 3 is a major kinase-independent target of sorafenib in hepatocellular carcinoma. J Hepatol. 2011;55:1041-8.

25. Huynh H, Nguyen TT, Chow KH, Tan PH, Soo KC, Tran E. Over-expression of the mitogen-activated protein kinase (MAPK) kinase (MEK)-MAPK in hepatocellular carcinoma: its role in tumor progression and apoptosis. BMC Gastroenterol. 2003;3:19. 
26. He G, Yu GY, Temkin V, Ogata H, Kuntzen C, Sakurai T, et al. Hepatocyte IKKbeta/ NF-kappaB inhibits tumor promotion and progression by preventing oxidative stress-driven STAT3 activation. Cancer Cell. 2010;17:286-97.

27. Sertel S, Eichhorn T, Simon $\mathrm{CH}$, Plinkert PK, Johnson SW, Efferth T. Pharmacogenomic identification of c-Myc/Max-regulated genes associated with cytotoxicity of artesunate towards human colon, ovarian and lung cancer cell lines. Molecules. 2010;15:2886-910.

28. Zhang Y, Xu G, Zhang S, Wang D, Saravana Prabha P, Zuo Z. Antitumor research on artemisinin and its bioactive derivatives. Nat Prod Bioprospect. 2018;8:303-19.

29. Finkel T. Signal transduction by reactive oxygen species. J Cell Biol. 2011; 194:7-15.
30. Khan Al, Kapoor A, Che J, Martin L, Rogazzo M, Mercier T, et al. The anti-malarial drug artesunate attenuates cardiac injury in a rodent model of myocardial infarction. Shock 2017. https://doi.org/10.1097/SHK.0000000000000963.

31. Schieber M, Chandel NS. ROS function in redox signaling and oxidative stress. Curr Biol. 2014;24:R453-62.

32. Kundu J, Kim DH, Chae IG, Lee JK, Lee S, Jeong CH, et al. Silicon dioxide nanoparticles induce COX-2 expression through activation of STAT3 signaling pathway in HaCaT cells. Toxicol Vitr. 2018;52:235-42.

33. Bae YS, Kang SW, Seo MS, Baines IC, Tekle E, Chock PB, et al. Epidermal growth factor (EGF)-induced generation of hydrogen peroxide. Role in EGF receptormediated tyrosine phosphorylation. J Biol Chem. 1997;272:217-21. 